\title{
Motivación deportiva en jugadoras de baloncesto de alto rendimiento de las selecciones nacionales de Argentina, Chile, Colombia, Ecuador, Paraguay, Perú y Venezuela
}

Sport motivation in high-performance basketball female players of national teams from Argentina, Chile, Colombia, Ecuador, Paraguay, Perú y Venezuela

Motivação esportiva em jogadores de basquete de alto rendimento das seleções da Argentina, Chile, Colombia, Ecuador, Paraguay,

Peru e Venezuela

\section{Liliana Paola Muñoz Gómez ${ }^{1}$ Solbey Morillo Puente ${ }^{2}$ Edwin Eduardo Hernández Bohórquez ${ }^{3}$}

Recibido: 22 de abril de 2020 Aprobado: 7 de diciembre de 2020

Publicado: 12 de enero de 2021

Cómo citar este artículo: Muñoz Gómez, L. P., Morillo Puente, S. y Hernández Bohórquez, E. E. (2020). Motivación deportiva en jugadoras de baloncesto de alto rendimiento de las selecciones nacionales de Argentina, Chile, Colombia, Ecuador, Paraguay, Perú y Venezuela.

Pensando Psicología, 17(1), 1-26. doi: https://doi.org/10.16925/2382-3984.2021.01.04

Artículo de investigación. https://doi.org/10.16925/2382-3984.2021.01.04

1 Universidad de Medellín

ORCID: https://orcid.org/0000-0001-8995-7404

Correo electrónico: lilipao2014@gmail.com

2 Universidad de Medellín

ORCID: https://orcid.org/0000-0002-2129-1121

3 Universidad Pedagógica y Tecnológica de Colombia

ORCID: https://orcid.org/0000-0002-2016-1480 
2 Motivación deportiva en jugadoras de baloncesto de alto rendimiento de las selecciones nacionales de Argentina, Chile, Colombia, Ecuador, Paraguay, Perú y Venezuela

\section{Resumen}

Objetivo: analizar los niveles de motivación desde la motivación intrínseca, extrínseca y la no motivación en 83 mujeres entre los 18 y 23 años de edad que pertenecen a la selección nacional de baloncesto de siete países latinoamericanos.

Metodología: a partir de la recolección de los datos se llevó a cabo una investigación observacional, transeccional y correlacional, en la cual se suministró la escala de motivación deportiva (SMS/EMD de Pelletier, et al., 1995). Los resultados más representativos muestran que el $82,7 \%$ y $43,4 \%$ de las participantes presentaron niveles altos de motivación, intrínseca y extrínseca respectivamente.

Resultados: los niveles de motivación intrínseca difieren según los países, pero no de manera estadísticamente significativa, mientras que la motivación extrínseca si varió de manera significativa entre países.

Conclusiones: se identificó que un 13,4 \% de no motivación está presente en las jugadoras y que no hay diferencias significativas entre países.

Palabras clave: América latina, deporte, estadística y juego.

\section{Abstract}

Aim: To analyze levels of extrinsic, intrinsic, and non-motivation in 83 women between 18 and 23 years of age who belong to seven basketball national teams of seven Latin American countries.

Methodology: From the data collection, an observational, cross-sectional, and correlational research was carried out, in which the Sport Motivation Scale (Pelletier et al., 1995) was applied. The most representative results show that $82.7 \%$ and $43.4 \%$ of participants presented high levels of motivation, intrinsic and extrinsic respectively.

Results: Levels of intrinsic motivation differ according to the countries, but not in a statistically significant way, while the extrinsic motivation did vary significantly between countries.

Conclusions: It was identified that $13.4 \%$ of non-motivation is present in the players and that there are no significant differences between countries.

Keywords: Latin America, sport, statistics, play.

\section{Resumo}

Objetivo: Analisar os níveis de motivação, a partir da motivação intrínseca, extrínseca e desmotivação, em 83 mulheres entre os 18 e 23 anos de idade pertencentes à seleção nacional de basquete de sete países latino-americanos.

Metodologia: A partir da coleta de dados, foi realizada uma pesquisa observacional, transversal e correlacional, na qual forneceu a escala de motivação esportiva (SMS / EMD de Pelletier, et al., 1995). Os resultados mais representativos mostraram que $82,7 \%$ e $43,4 \%$ das participantes apresentaram níveis elevados de motivação, intrínseca e extrínseca, respectivamente.

Resultados: Os níveis de motivação intrínseca diferem de acordo com os países, mas não de forma estatisticamente significativa, enquanto a motivação extrínseca varia significativamente entre os países.

Conclusões: Identificou-se que 13,4\% de não motivação está presente nas jogadoras e que não há diferenças significativas entre os países.

Palavras-chave: América latina, esporte, estatística, jogo. 


\section{Introducción}

La motivación constituye un elemento fundamental en el trabajo con deportistas, pues orienta la conducta y le da sentido a la acción; según (Buceta, 1999a), se trata de un factor determinante que considera un conjunto de variables que intervienen en la elección de la intensidad de la práctica y la persistencia en la tarea en cuanto al rendimiento deportivo. Los deportistas tienen niveles elevados de motivación cuando así lo deciden; sin embargo, existen otros factores que además pueden afectar o influir en el rendimiento, tales como los padres, los compañeros e incluso los directivos (García et al., 2014) y el entrenador (Pulido, et al., 2016).

Para Dosil y Caracuel (2003), la motivación es una especie de motor que dirige la acción al máximo hacia el logro de metas; es una variable psicológica que, para estos autores, estabiliza y le da carácter a la acción deportiva. En este sentido, en el ámbito deportivo la motivación ha sido uno de los procesos más estudiados por los expertos, y se asocia con la iniciación, mantenimiento y abandono de la práctica del deporte, además, la adaptación deportiva se supera cuando existen niveles elevados de motivación (Guillén, 2007).

La evaluación de la motivación en el deporte es de vital importancia, los expertos del tema se han interesado por valorar aspectos como: diferentes características psicológicas, niveles de competitividad, el disfrute del deporte, el mejoramiento de las técnicas deportivas, la resolución de problemas en el campo de juego, el interés y el esfuerzo (Guzmán-Luján y Carratalá-Deval, 2006).

Es de resaltar que a nivel mundial, existen investigaciones sobre la motivación en el campo deportivo que muestran resultados obtenidos en diversos grupos poblacionales y diferentes deportes; en este estudio fue primordial analizar los niveles de motivación deportiva en basquetbolistas de los equipos nacionales sudamericanos 2018, el grupo poblacional estuvo compuesto por jugadoras activas pertenecientes a las selecciones nacionales de baloncesto femenino de Chile, Colombia, Ecuador, Paraguay, Perú y Venezuela, quienes participaron en un encuentro en Colombia en octubre del año 2018.

Para Blanco y Buceta (1993), el rendimiento deportivo en general, es cuestión de identificar e intervenir gradualmente hacia el logro de la meta, pero, como lo expresan González y Ríos (1999), la motivación individual en las mujeres está relacionada con el ámbito familiar, la emancipación femenina y la evitación de otros deberes que estimulan el gusto por lo que se hace. Otras categorías que se pueden fomentar la motivación deportiva giran en torno: viajes, independencia y valoración hacia la admiración (García-Sandoval y Caracuel-Tubio, 2007). 
4 Motivación deportiva en jugadoras de baloncesto de alto rendimiento de las selecciones nacionales de Argentina, Chile, Colombia, Ecuador, Paraguay, Perú y Venezuela

Las teorías de la motivación varían de acuerdo con los diversos autores, pero para este estudio se tuvieron en cuenta los postulados de Pelletier et al. (1995) y Balaguer et al. (2007), quienes definen tres factores motivacionales: la motivación intrínseca (MI), la motivación extrínseca (ME) y la no motivación (NM). La MI está conformada por: motivación para conocer, experimentar estimulación y conseguir cosas. La ME está constituida por: la regulación identificada, introyectada y externa. Y, finalmente, la NM no cuenta con subfactores (Cox, 2009).

Según Gutiérrez (2000), los deportistas tienden a perder la MI, considerada como la más potente, estable y duradera. Como lo afırman De Diego y Sagredo (1992), la MI está constituida por autorrecompensas; la ME en cambio, se regula dependiendo de los factores externos del jugador o jugadora. Otros estudios señalan que las motivaciones para los que practican deportes son la competición, la capacidad personal y la aventura (Moreno et al., 2005).

Por otro lado, se han estudiado diferentes tipos de motivación desde la motivación intrínseca y extrínseca en diferentes edades y géneros, así como aquellos que dedican más o menos días a entrenamiento (Moreno et al., 2006). Así mismo, el desarrollo emocional, motivacional y social de los deportistas de alto nivel pueden llegar a ser contradictorios (Gotzent y González, 1993).

Adicionalmente, la MI puede llegar a ser suficiente para contrarrestar a la ME, lo que implica que el emprendimiento y la persistencia juegan un papel importante en la acción. Dentro de los factores extrínsecos, a partir de (Bakhsh, 2008), se encuentran como significantes para la mujer: el buen trato, la seguridad, el ambiente laboral, la estabilidad y el reconocimiento.

A partir de Balaguer et al. (2007), el modelo teórico sobre el cual se basó este estudio implica el análisis psicométrico de la estructura factorial de una versión española de la escala de motivación deportiva de Deci y Ryan (1990), a partir de siete factores en los que existe una consistencia interna interesante. Los modelos se ajustaron para el habla hispana, razón por la cual se usa la escala adaptada y no la original, las correlaciones que se pueden encontrar entre los tres niveles de motivación orientan la estructura que regula la motivación deportiva en los niveles motivación extrínseca, motivación intrínseca y no motivación.

La escala de motivación deportiva (EMD), adaptada por Pelletier et al. (1995), reconoce tres niveles dentro de los cuales se estudian indicadores que los conforman: motivación intrínseca MI para conocer, MI para experimentar estimulación y MI para conseguir las cosas; motivación extrínseca: ME de regulación identificada, ME de regulación introyectada y ME regulación externa, y finalmente la no motivación. En consecuencia, las correlaciones que se pueden hallar entre los tres niveles 
de motivación dan cuenta de la existencia o inexistencia de los indicadores que cada subescala contiene. Esto a su vez implica que la motivación a partir de esta teoría, pone de manifiesto que los modelos alternativos pueden ser hipotetizados para luego analizarlos, se podría llegar a creer que existe la tendencia a encontrar una significación marcada en alguno de los tres niveles de motivación que entre sí, pueden llegar incluso a ser diferenciadores mas no integrales en los deportistas; es decir, que existe distintas formas de regular la motivación a partir de la presencia de una de ellas y no de los tres niveles al mismo tiempo (Balaguer et al., 2007).

Por otra parte, el abandono en el deporte puede ser cuestión de desánimo o no motivación en la participación deportiva de los jóvenes. Ahora bien, circunstancias adversas como conflictos de intereses, demasiados deportistas, pocos estudios y problemas metodológicos dificultan determinar los factores reales que influyen en la decisión de mantenerse o desertar del deporte que se practica (Puig, 1992), aunque también se puede relacionar negativamente con la desmotivación (Pulido et al., 2015).

A nivel educativo, se ha estudiado la motivación para entender la deserción universitaria, lo que ha permitido identificar relaciones entre la motivación académica, la procrastinación y los estilos de afrontamiento (Montgomery et al., 2017). También para explicar el comportamiento lector, Mújica et al. (2012) establecen la existencia de una disposición motivacional en la conducta lectora.

En cuanto al entrenador, los autores encuentran que los deportistas cuyos entrenadores carecen de formación federativa presentan mayores niveles de desmotivación, de allí que destaquen la importancia de la formación de este esencial componente de un equipo. La motivación en el sujeto mantiene el interés, y la práctica deportiva, por el contrario, puede intervenir de manera negativa hacia el abandono del juego. Otros factores que se encuentran relacionados con la motivación, según Escartí y Cervelló (1994), son biológicos, emocionales, sociales y cognitivos. Entre los biológicos se encuentran el sexo, la edad, el sobrepeso y el historial de lesiones (Flores y Ruiz, 2009).

A nivel académico, otras investigaciones, como la de González et al. (2018), por ejemplo, recomiendan que para el análisis del rendimiento académico de los alumnos se incluyan indicadores psicológicos tanto de bienestar como de desajuste psicológico, entre ellos; el nivel de autoestima, autoeficacia y motivación, reconociendo que las poblaciones no son homogéneas en características intelectuales, emocionales y motivacionales. Afirman, que el logro de rendimiento excepcional requiere altos niveles de motivación asociados a la capacidad de aprendizaje y creatividad.

En cuanto al género, Cruz (1997) afirma que las mujeres que compiten en el baloncesto profesional requieren de un esfuerzo y un aprendizaje superior que regule 
6 Motivación deportiva en jugadoras de baloncesto de alto rendimiento de las selecciones nacionales de Argentina, Chile, Colombia, Ecuador, Paraguay, Perú y Venezuela

su práctica, lo cual surge básicamente de la motivación intrínseca, esto determina en efecto el rendimiento deportivo de la jugadora, lo que significa que si está altamente motivada está en capacidad de rendir favorablemente en el deporte. Esta aseveración es secundada por Giménez (2004) y Buceta (1999b), para quienes el baloncesto no se juega de manera impersonal, ya que la motivación es influyente en la acción de la conducta, lo que significa que las jugadoras necesitan estar motivadas para competir; la motivación ayuda entonces al mejoramiento del bienestar y satisfacción no solo individual sino grupal, lo que implica que debe ser estudiada tanto personal como colectivamente en la psicología deportiva (Valdés, 1990).

Al parecer las mujeres se desmotivan menos que los hombres, debido a un mejor manejo de la ansiedad, estrés y depresión (Muyor et al., 2009). En este sentido, la motivación femenina constituye la productividad del momento, es un factor que canaliza diversos recursos, apuntando a una mayor participación y parece estar ligada a la independencia y el equilibrio (Alfaro, 2010). La autonomía, por ejemplo, generalmente orienta de manera interdependiente a la MI, en tanto que el reconocimiento implica la percepción hacia el trabajo arduo en la mujer, la motivación se asocia más a factores de tipo personal que a aspectos de satisfacción extrínseca (Huilcapi et al., 2017).

En síntesis, la motivación está determinada, igualmente, por factores sociales como autonomía, control, estilos de enseñanza y, en consecuencia, por la satisfacción de las necesidades humanas fundamentales de competencia, autonomía y relación con los demás (Broo, et al., 2012); en este sentido se destaca el rol del contexto social en la motivación, al igual que la calidad de vida está influenciada por la motivación (Gómez, 2019).

Finalmente, la pretensión de este trabajo se enfoca en la psicología deportiva y cómo la motivación se hace presente en jugadoras de baloncesto profesional en siete equipos de Latinoamérica desde una revisión estadística a partir del suministro de una escala que permite identificar tres tipos de motivación: MI, ME y NM.

\section{Método}

\section{Tipo de estudio}

Se diseñó un estudio de tipo observacional, descriptivo, transversal y correlacional a través de una prueba tipo autoinforme aplicado a una muestra probabilística (Hernández et al., 2014). El objetivo del estudio fue analizar los niveles de motivación desde la motivación intrínseca, extrínseca y la no motivación en 83 mujeres entre los 18 y 23 años que pertenecen a la selección nacional de baloncesto de siete países 
latinoamericanos mediante una investigación observacional, transeccional y correlacional, en la cual se suministró la escala de motivación deportiva SMS/EMD de Pelletier et al. (1995). Las hipótesis que guiaron este estudio fueron: existen diferencias estadísticas significativas en los niveles de motivación en 83 mujeres entre los 18 y 23 años de las selecciones nacionales de baloncesto de siete países latinoamericanos. No existen diferencias estadísticamentes significativas en los niveles de motivación en 83 mujeres entre los 18 y 23 años de las selecciones nacionales de baloncesto de siete países latinoamericanos.

\section{Participantes}

Se llevó a cabo un muestreo al azar simple (Montero y León, 2007), en el que se incluyó la totalidad de las jugadoras de habla hispana de siete equipos de baloncesto profesional que participaron en el Encuentro Sudamericano Femenino en Colombia, en agosto de 2018, con el objeto de analizar los niveles de motivación deportiva. La muestra estuvo constituida por 83 mujeres entre los 18 y 23 años de edad correspondientes a siete equipos de alto rendimiento: Argentina, Chile, Colombia, Ecuador, Paraguay, Perú y Venezuela (tabla 1). Los criterios de exclusión que se tuvieron en cuenta fue la totalidad de los equipos que participaron de la convocatoria deportiva para la fecha en mención; la encuesta la respondieron la totalidad de las jugadoras que pertenecían a los siete equipos en competencia, a excepción de Brasil, debido a la diferencia de idioma.

Tabla 1. Muestra de deportistas de alto rendimiento en baloncesto

\begin{tabular}{|c|c|c|}
\hline País & $\mathbf{N}^{\circ}$ de jugadoras & \% jugadoras \\
\hline Argentina & 12 & 14,5 \\
\hline Chile & 12 & 14,5 \\
\hline Colombia & 12 & 14,5 \\
\hline Ecuador & 12 & 14,5 \\
\hline Paraguay & 11 & 13,3 \\
\hline Perú & 13 & 15,7 \\
\hline Venezuela & 11 & 13,3 \\
\hline Total & 83 & 100,0 \\
\hline
\end{tabular}

Fuente: los autores. 
8 Motivación deportiva en jugadoras de baloncesto de alto rendimiento de las selecciones nacionales de Argentina, Chile, Colombia, Ecuador, Paraguay, Perú y Venezuela

\section{Instrumentos}

Se aplicó una prueba validado, revisado y adaptado, denominada "escala de motivación deportiva (EMD)" (Pelletier et al., 1995), la cual está formada por tres factores: motivación intrínseca, extrínseca y no motivación. Esta prueba es el resultado del análisis psicométrico convertido a la versión en habla hispana.

\section{Escala de motivación deportiva (EMD)}

La prueba de motivación deportiva (EMD) consiste en una prueba adaptada por Pelletier et al. (1995), es un autoinforme de 28 ítems que mide: (motivación intrínseca): Ml para conocer, Ml para experimentar estimulación y MI para conseguir las cosas; (motivación extrínseca): ME de regulación identificada, ME de regulación introyectada y ME regulación externa, y, finalmente, la (no motivación).

Esta prueba (EMD) orienta al deportista a responder una serie de ítems sobre la premisa: ¿por qué participas en tu deporte? En el cual debe elegir de 1 a 7 con tres subescalas de medición: no tiene nada que ver conmigo, tiene algo que ver conmigo y se ajusta totalmente a mí. La prueba se suministró de manera colectiva en cada uno de los equipos profesionales y en forma individual para cada deportista. La prueba tiene una validez alta entre poblaciones de habla hispana (Balaguer et al., 2007).

\section{Procedimiento}

Se aplicaron las pruebas en la liga deportiva de Tunja (Colombia) durante el Encuentro Sudamericano Femenino en Colombia, en el año 2018. Se contactó a los entrenadores de los siete países seleccionados. La duración del diligenciamiento de la prueba osciló entre los 20 y 40 minutos.

\section{Consideraciones éticas}

Los participantes firmaron un consentimiento informado autorizando el empleo de los datos de manera confidencial para la investigación y futuras publicaciones. Se indicó a los participantes que podían realizar las preguntas que surgieran durante la aplicación y que se podían retirar en cualquier momento si así lo consideraban pertinente. Con relación a la declaración de Helsinki, se tuvo en cuenta los requerimientos éticos, entre los cuales se señala: se veló por el bienestar de los derechos de los participantes, la investigación tuvo en cuenta las normas éticas para la promoción y aseguramiento del respeto de quienes hicieron parte de este estudio, se mantuvo la 
confidencialidad del manejo de los datos, se contemplaron y controlaron los riesgos de los seres humanos en cuestión, se hizo un procedimiento cuidadoso del manejo de la información y, finalmente, se redujeron al máximo los riesgos que pudiera acarrear esta investigación en las personas objeto de estudio. El consentimiento informado se hizo mediante protocolo de investigación, en donde los participantes conocían sus derechos y estaban enterados del procedimiento voluntario y la posibilidad de retirarse cuando así lo consideraran necesario.

\section{Técnicas de análisis}

Después de aplicada la prueba, tomando en consideración los lineamientos de la prueba, se procedió al análisis estadístico mediante una codificación de la información y se analizaron los datos basados en los objetivos del estudio, con la ayuda del programa SPSS v.22.

En primera medida, se hizo un análisis descriptivo de los niveles de motivación intrínseca, extrínseca y no motivación de las deportistas empleando frecuencias absolutas y porcentajes. Posteriormente, se determinó la existencia de relación entre los niveles de motivación y el país al que representaban las jugadoras mediante la prueba chi cuadrado de Pearson, a un nivel de significación del $5 \%(a=0,05)$.

En este estudio se empleó el instrumento traducido al habla hispana por Pelletier et al. (1995), el cual contiene 28 reactivos, que fueron el único interés que guio este trabajo investigativo, las demás escalas no fueron tenidas en cuenta ni se hizo un análisis comparativo, debido a que no cumplían con los criterios de inclusión para la recolección y análisis de los datos conforme con las características de la muestra. Estos 28 reactivos se analizaron por niveles y subescalas de manera individualizada, el número de reactivos para cada subnivel que no altera los resultados debido a que el análisis así fue guiado.

La recogida de los datos estuvo a cargo de uno de los entrenadores, y todas las deportistas aceptaron participar de manera voluntaria en el estudio. Una vez se recogieron los datos, fueron tabulados y analizados conforme indican los autores de la escala para el estudio de los reactivos. Se hizo un análisis estadístico amplio por niveles, subescalas, reactivos y equipos deportivos. Se empleó el programa estadístico SPSS para realizar las pruebas de chi cuadrado de independencia para establecer la relación entre las variables.

Los parámetros estimados para este estudio respetaron la guía de análisis que propone la escala adaptada por Pelletier et al. (1995), la cual contiene modificaciones de análisis estadístico con relación a la escala original de (Deci y Ryan, 1990). Los 
índices de los demás modelos de la escala no fueron tenidos en cuenta, se analizó la totalidad de los datos a partir del propósito del modelo alternativo que nos brinda la adaptación de la escala. El interés se enmarcó en reconocer las diferencias estadísticamente significativas, a partir de la relación entre las variables, desde una estructura factorial de siete subescalas en siete equipos de baloncesto femenino suramericanos.

\section{Resultados}

Los resultados de la prueba de motivación deportiva (SMS/EMD) se presentan a continuación. Con relación al objetivo de estudio general -analizar los niveles de motivación deportiva en basquetbolistas de los equipos nacionales sudamericanos 2018- específicamente se analizaron: 1. niveles de MI, 2. niveles de ME y 3. niveles de NM, de manera separada. Para ello se tomaron las respuestas de los ítems que componen la prueba y sus dimensiones y se clasificaron siguiendo el criterio establecido por Pelletier et al. (1995).

En este orden de ideas, cada dimensión de motivación se determinó a partir del promedio de cuatro ítems, cuyas respuestas estaban en una prueba de 1 a 7 puntos, donde 1 y 2 indicaban "no tiene nada que ver conmigo", 3-5 "tiene algo que ver conmigo" y 6-7 "se ajusta totalmente a mí". Estos resultados se reagruparon para formar tres niveles de motivación: motivación baja (1-3 puntos), motivación media (3-5 puntos) y motivación alta (5-7 puntos).

Se exploraron las tres dimensiones de MI. Los resultados de las 83 jugadoras de baloncesto muestran que, en este grupo de deportistas, la mayoría está altamente motivada, tanto en MI para conocer (80,1 \%) como para experimentar estimulación (92,7 \%) y para conseguir cosas $(81,9 \%)$, destacando la $\mathrm{Ml}$ para experimentar estimulación, en la que casi todas tienen alta motivación. Esto indica que de cada diez jugadoras de baloncesto al menos ocho tiene alta motivación intrínseca.

Los resultados revelan que es muy bajo el porcentaje de basquetbolistas con baja motivación, siendo el porcentaje más pequeño el de la MI para conocer (2,4 \%), en tanto que el nivel bajo de MI para conseguir cosas fue de 3,6 \%. Los porcentajes de MI total develan una gran proporción de alta motivación (82,7 \%) y un nivel bajo casi inexistente (1,2\%) (tabla 2). 
Tabla 2. Niveles de motivación intrínseca de las jugadoras de baloncesto. Bogotá, 2018

\begin{tabular}{|c|c|c|c|c|c|c|c|}
\hline \multirow{3}{*}{ Motivación intrínseca } & \multicolumn{6}{|c|}{ Nivel de motivación intrínseca } & \multirow{3}{*}{$\mathbf{n}$} \\
\hline & \multicolumn{2}{|c|}{ Nivel bajo } & \multicolumn{2}{|c|}{ Nivel medio } & \multicolumn{2}{|c|}{ Nivel alto } & \\
\hline & $\mathbf{N}^{\circ}$ & $\%$ & $\mathbf{N}^{\circ}$ & $\%$ & $\mathbf{N}^{\circ}$ & $\%$ & \\
\hline Para conocer & 2 & 2,4 & 14 & 17,1 & 66 & 80,1 & 82 \\
\hline Para experimentar estimulación & 1 & 1,2 & 5 & 6,1 & 76 & 92,7 & 82 \\
\hline Para conseguir cosas & 3 & 3,6 & 12 & 14,5 & 68 & 81,9 & 83 \\
\hline Total & 1 & 1,2 & 13 & 16,0 & 67 & 82,7 & 81 \\
\hline
\end{tabular}

Fuente: los autores.

Las tres dimensiones de motivación extrínseca y sus niveles se representan en la figura 1. Se puede apreciar que la proporción de las jugadoras que presentan niveles bajos de ME regulación identificada es apenas del $6 \%$, y que este porcentaje es más alto en regulación introyectada (20,5\%); en regulación externa, 2 de cada 10 deportistas tiene un bajo nivel de motivación. A diferencia de la Ml en la que la mayoría tenía niveles altos de motivación. En la ME los niveles altos no superan el 70 \%; así, en la dimensión regulación identificada que mostró los mayores porcentajes de alta motivación, se registró que el 66,3 \% de las jugadoras de baloncesto tienen un alto nivel de motivación, en tanto que en regulación introyectada y regulación externa estos porcentajes estuvieron por debajo el $50 \%$ (47\% y $41 \%$, respectivamente). El puntaje total de motivación extrínseca indica que, de cada diez jugadoras de baloncesto, cuatro tienen niveles de motivación alto, cuatro nivel medio y dos están en nivel bajo de motivación.

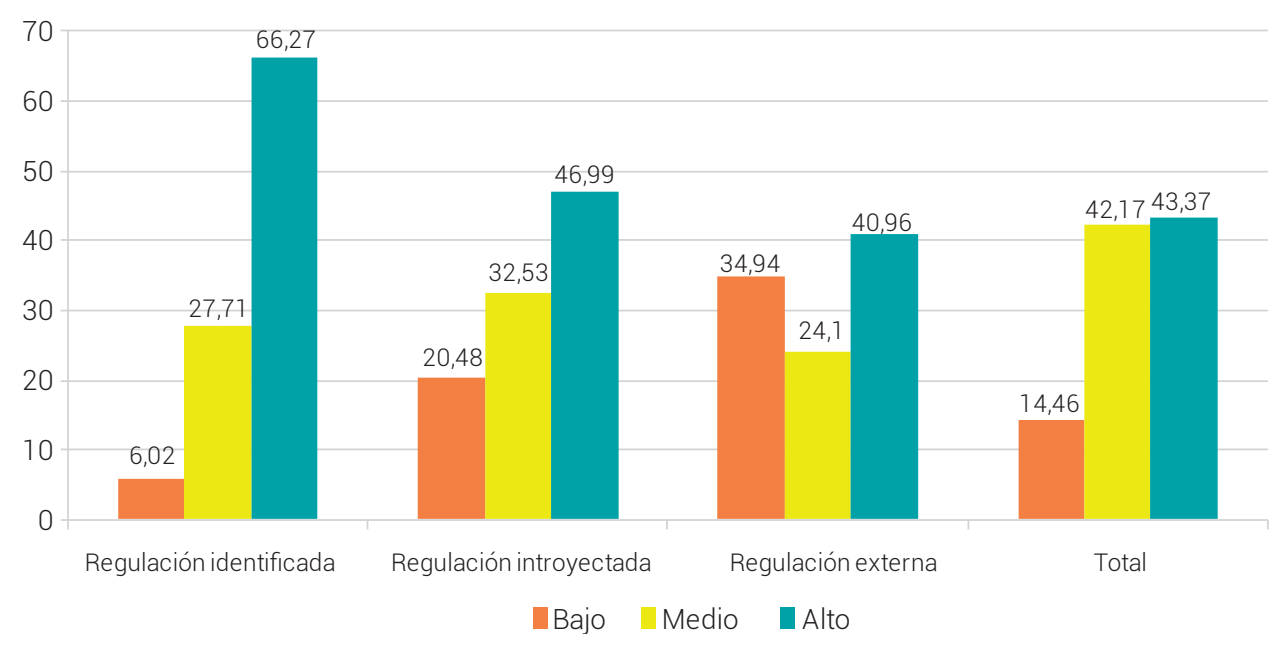

Figura 1. Niveles de motivación extrínseca de las jugadoras de baloncesto. Bogotá, 2018 
La no motivación es el promedio de cuatro ítems que indagaban en torno al deporte. Aspectos como: "Solía tener buenas razones para practicar este deporte, pero actualmente me pregunto si debería continuar haciéndolo" Pelletier et al. (1995), develan que la basquetbolista no tiene motivación para participar en la disciplina deportiva. Estas puntuaciones se agruparon tomando como criterio una unidad por encima del promedio, que en este caso corresponde a puntajes por encima de 4,1 puntos y menores o iguales a 4 puntos. Esta categorización llevó a clasificar un 86,6 \% de las deportistas en el grupo de no motivación ausente, es decir, que son jugadoras que en su mayoría carecen de no motivación. Estos resultados que se presentan en la tabla 4 son consistentes con los de alta MI (82,7\%) en estas mismas basquetbolistas. Dicho de otro modo, de cada diez jugadoras de baloncesto, menos de dos participantes tienen no motivación (tabla 3).

Tabla 3. Niveles de no motivación de las jugadoras de baloncesto. Bogotá, 2018

\begin{tabular}{lcc}
\hline No motivación & $\mathbf{N}^{\circ}$ de jugadoras & \% \\
\hline Ausente & 71 & 86,6 \\
\hline Presente & 11 & 13,4 \\
\hline Total & 82 & 100,0 \\
\hline
\end{tabular}

Fuente: los autores.

Como se indicó, las deportistas pertenecían a las selecciones nacionales de siete países, partícipes de los juegos sudamericanos para el año 2018. Se puede observar en la figura 2 que solo dos países (Colombia y Paraguay) tenían jugadoras cuyo subnivel de motivación intrínseca denominado MI para conocer, corresponde al nivel bajo. Destaca también que todas las jugadoras de Chile tienen un alto nivel de motivación, seguido en porcentaje por Perú (92,3 \%) y Venezuela (90 \%). El 58,3 \% de las jugadoras del equipo de Argentina tiene un nivel de motivación Alto. En Ecuador, tres de cada cuatro jugadoras tiene alto nivel de motivación intrínseca. 


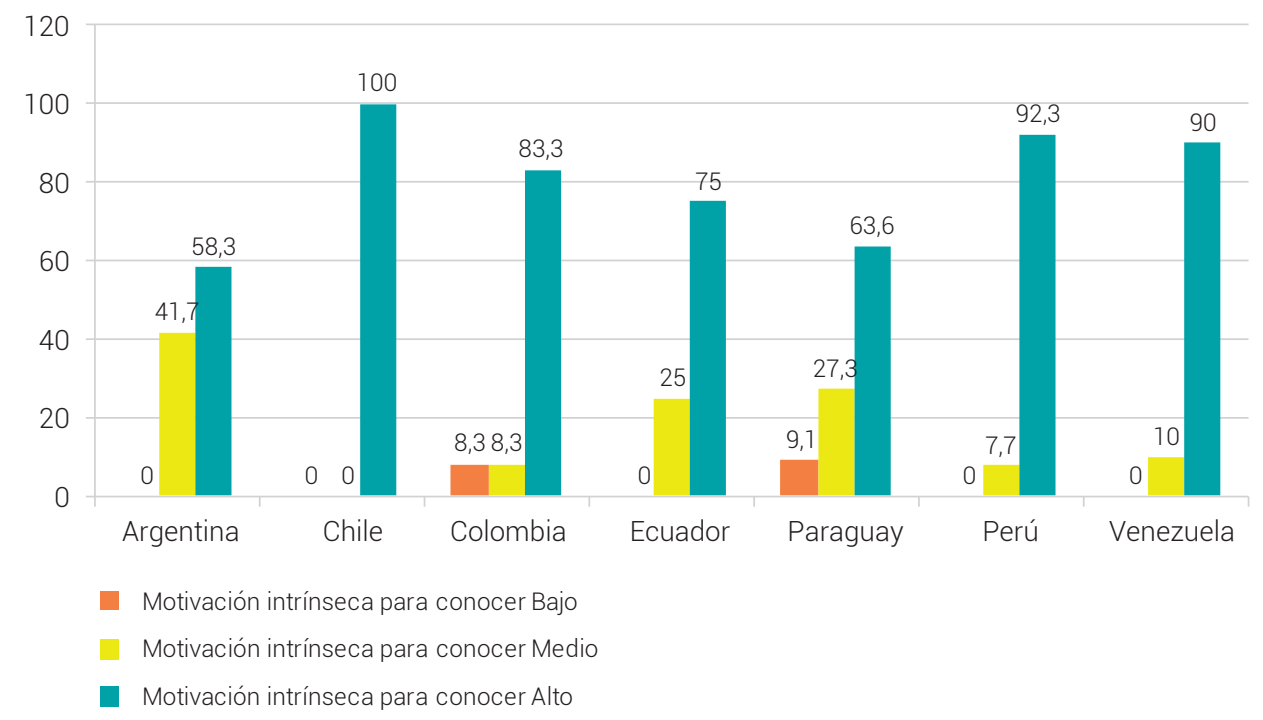

Figura 2. Niveles de motivación intrínseca para conocer según país de las jugadoras de baloncesto. Bogotá, 2018

Los resultados del subnivel (MI para experimentar estimulación) se resumen en la tabla 4, en la que destaca que todas las jugadoras de Argentina, Chile y Venezuela exhiben un nivel alto de esta dimensión, en tanto que Colombia tuvo el menor porcentaje de jugadoras en este nivel (75 \%), lo que muestra que tres de cada cuatro jugadoras tiene una motivación alta. Paraguay, Ecuador y Perú también tienen altos porcentajes de jugadoras con altos niveles de motivación. El único país que tiene una jugadora con un nivel bajo de motivación extrínseca es Colombia.

Tabla 4. Niveles de motivación intrínseca para experimentar estimulación según País de las jugadoras de baloncesto. Bogotá, 2018

\begin{tabular}{|c|c|c|c|c|c|c|c|}
\hline \multirow{3}{*}{ País } & \multicolumn{6}{|c|}{ Nivel de motivación intrínseca para experimentar estimulación } & \multirow{3}{*}{$\mathbf{n}$} \\
\hline & \multicolumn{2}{|c|}{ Nivel bajo } & \multicolumn{2}{|c|}{ Nivel medio } & \multicolumn{2}{|c|}{ Nivel alto } & \\
\hline & $\mathbf{N}^{\circ}$ & $\%$ & $\mathbf{N}^{\circ}$ & $\%$ & $\mathbf{N}^{\circ}$ & $\%$ & \\
\hline Argentina & 0 & -- & 0 & -- & 12 & 100,0 & 12 \\
\hline Chile & 0 & -- & 0 & -- & 12 & 100,0 & 12 \\
\hline Colombia & 1 & 8,3 & 2 & 16,7 & 9 & 75,0 & 12 \\
\hline Ecuador & 0 & -- & 1 & 8,3 & 11 & 91,7 & 12 \\
\hline Paraguay & 0 & -- & 1 & 9,1 & 10 & 90,0 & 11 \\
\hline Perú & 0 & -- & 1 & 8,3 & 11 & 91,7 & 12 \\
\hline Venezuela & 0 & -- & 0 & -- & 11 & 100,0 & 11 \\
\hline
\end{tabular}

Fuente: los autores. 
En la MI para conseguir cosas, como puede observarse en la figura 3, Chile es el único país cuyas jugadoras en su totalidad tienen un alto nivel de motivación intrínseca para conseguir cosas, en tanto que solo la mitad de las jugadoras de Argentina tiene dicho nivel de motivación. De cada cuatro jugadoras de Colombia, tres tienen alto nivel de motivación intrínseca para conseguir cosas. Los niveles bajos de esta motivación se registran en baja proporción solo para Argentina, Colombia y Paraguay con una sola jugadora en este nivel.

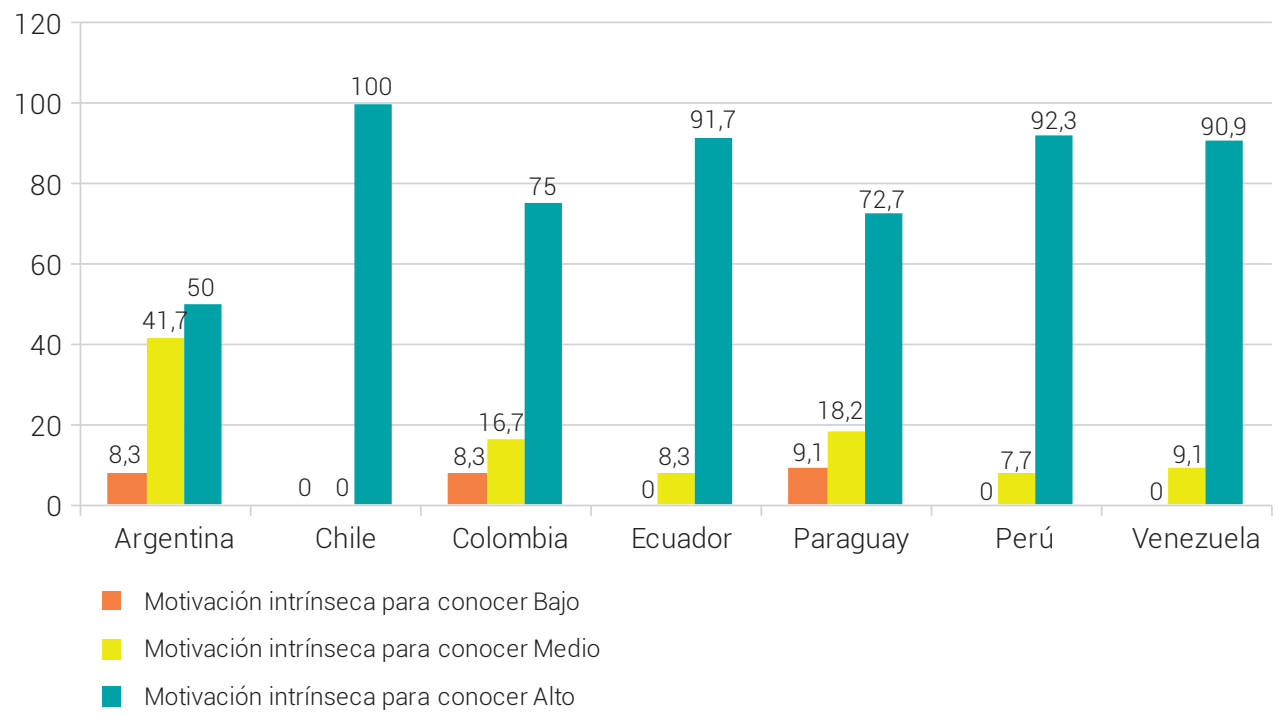

Figura 3. Niveles de motivación intrínseca para conseguir cosas según país de las jugadoras de baloncesto. Bogota, 2018

Respecto a la ME regulación identificada, los resultados de la tabla 5 destacan que todas las jugadoras de Venezuela tienen un nivel alto, seguido por Chile donde casi la totalidad de su equipo también tiene un alto nivel (9,7\%). Los demás países tuvieron menores porcentajes de alto nivel de motivaciones y, de estos, Argentina y Colombia son los que tienen los valores más bajos, incluso por debajo de la mitad (41,7 \%). También debe resaltarse que Paraguay y Venezuela fueron los únicos países que no tuvieron jugadoras con bajo nivel de motivación extrínseca regulación identificada. 
Tabla 5. Niveles de motivación extrínseca regulación identificada según país de las jugadoras de baloncesto. Bogotá, 2018

\begin{tabular}{|c|c|c|c|c|c|c|c|}
\hline \multirow{3}{*}{ País } & \multicolumn{6}{|c|}{ Nivel de motivación extrínseca regulación identificada } & \multirow{3}{*}{$\mathbf{n}$} \\
\hline & \multicolumn{2}{|c|}{ Nivel bajo } & \multicolumn{2}{|c|}{ Nivel medio } & \multicolumn{2}{|c|}{ Nivel alto } & \\
\hline & $\mathbf{N}^{\circ}$ & $\%$ & $\mathbf{N}^{\circ}$ & $\%$ & $\mathbf{N}^{\circ}$ & $\%$ & \\
\hline Argentina & 1 & 8,3 & 6 & 50,0 & 5 & 41,7 & 12 \\
\hline Chile & 1 & 8,3 & 0 & -- & 11 & 91,7 & 12 \\
\hline Colombia & 1 & 8,3 & 6 & 50,0 & 5 & 41,7 & 12 \\
\hline Ecuador & 1 & 8,3 & 2 & 16,7 & 9 & 75,0 & 12 \\
\hline Paraguay & 0 & -- & 5 & 45,5 & 6 & 54,5 & 11 \\
\hline Perú & 1 & 7,7 & 4 & 30,8 & 8 & 61,5 & 13 \\
\hline Venezuela & 0 & -- & 0 & -- & 11 & 100,0 & 11 \\
\hline
\end{tabular}

Fuente: los autores.

La ME de regulación introyectada que hace referencia a que el individuo comienza a internalizar las razones para sus acciones muestra que hay porcentajes más bajos en los niveles altos de motivación en las jugadoras de baloncesto. Es notorio, de manera particular, que Argentina solo tenga un $25 \%$ de sus jugadoras con altos niveles de motivación y que Chile que en casi todas las dimensiones de motivación tanto intrínseca como extrínseca tuvo la totalidad de sus jugadoras en nivel alto, en ME desde la regulación introyectada que hace referencia a la regulación de la conducta sin aceptarlas totalmente como propias, presenta ocho de cada diez jugadoras con alto nivel de motivación.

También llama la atención que Argentina, Paraguay y Perú tienen varias jugadoras con bajo nivel de motivación, cuando en las demás dimensiones de motivación se registraba apenas una jugadora o máximo dos en este nivel (tabla 6).

Tabla 6. Niveles de motivación extrínseca regulación introyectada según país de las jugadoras de baloncesto. Bogotá, 2018

\begin{tabular}{|c|c|c|c|c|c|c|c|}
\hline \multirow{3}{*}{ País } & \multicolumn{6}{|c|}{ Nivel de motivación extrínseca regulación introyectada } & \multirow{3}{*}{ n } \\
\hline & \multicolumn{2}{|c|}{ Nivel bajo } & \multicolumn{2}{|c|}{ Nivel medio } & \multicolumn{2}{|c|}{ Nivel alto } & \\
\hline & $\mathbf{N}^{\circ}$ & $\%$ & $\mathbf{N}^{\circ}$ & $\%$ & $\mathbf{N}^{\circ}$ & $\%$ & \\
\hline Argentina & 4 & 33,3 & 5 & 41,7 & 3 & 25,0 & 12 \\
\hline Chile & 1 & 8,3 & 1 & 8,3 & 10 & 83,3 & 12 \\
\hline Colombia & 3 & 25,0 & 4 & 33,3 & 5 & 41,7 & 12 \\
\hline Ecuador & 0 & -- & 7 & 58,3 & 5 & 41,7 & 12 \\
\hline Paraguay & 4 & 36,4 & 4 & 36,4 & 3 & 27,3 & 11 \\
\hline Perú & 4 & 30,8 & 3 & 23,1 & 6 & 46,2 & 13 \\
\hline Venezuela & 1 & 9,1 & 3 & 27,3 & 7 & 63,6 & 11 \\
\hline
\end{tabular}

Fuente: los autores. 
La ME desde la regulación externa indica que, entre las jugadoras de baloncesto, es notoria la presencia de niveles bajos de motivación en mayor proporción que en el resto de las motivaciones analizadas, siendo Argentina (50 \%), Perú (36,2 \%) y Paraguay (45,5\%) los países donde hay mayor porcentaje de jugadoras en el nivel bajo de motivación extrínseca regulación externa. Venezuela es el país con mayor cantidad de jugadoras cuyos niveles de motivación extrínseca son altos (72,7\%), seguido por Chile con un 66,7 \%. El resto de los países no llegó a tener más de la mitad de sus jugadoras con altos niveles de ME regulación externa, al tiempo que ningún país alcanzó a tener la totalidad de sus jugadoras con altos niveles de motivación (figura 4).

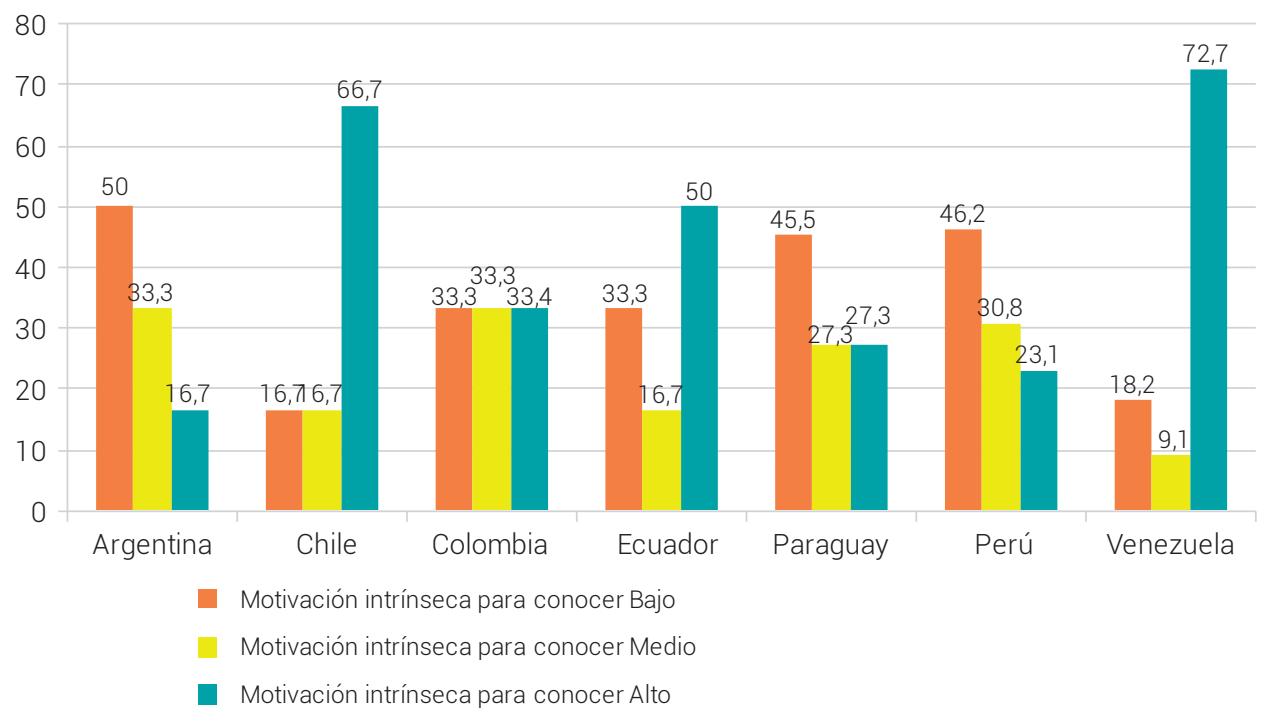

Figura 4. Niveles de motivación extrínseca regulación externa según país de las jugadoras de baloncesto. Bogotá, 2018

\section{Niveles de motivación intrínseca y extrínseca según país de las jugadoras}

Tal como se ha indicado, la mayoría de las jugadoras de baloncesto presentaron niveles altos de motivación. Por tal razón, se decidió agrupar los niveles de motivación bajo y medio para compararlos con el nivel alto de motivación y determinar si estos niveles tienen relación con el país al cual representaron las jugadoras en el Encuentro Sudamericano Femenino de 2018. La tabla 7 resume dichos niveles de motivación discriminados por países y en ella se puede apreciar que todas las basquetbolistas de la selección chilena tenían alta motivación intrínseca, seguidos por Perú y Venezuela en los cuales solo una de sus miembros mostró no tener alta motivación intrínseca. 
Argentina fue el país cuyas deportistas revelaron la menor proporción de alta motivación intrínseca (58,3\%). No obstante, estas diferencias observadas en las proporciones no son estadísticamente significativas, es decir, los niveles de motivación intrínseca no están asociados con el país. Con respecto a la motivación extrínseca, nuevamente Chile fue la selección que tuvo mayor porcentaje de jugadoras con niveles altos (75\%), seguido por Venezuela en el que seis de cada diez jugadoras tenían altos niveles de motivación. En el resto de los países el nivel de motivación extrínseca no alta fue lo que prevaleció, especialmente en Argentina (83,3 \%), Paraguay y Perú con $72,7 \%$ y 69,2 \%, respectivamente. Estas diferencias en las frecuencias de motivación extrínseca se deben a que las jugadoras pertenecen a diferentes equipos (países), los datos aportan suficiente evidencia para afirmar que existe relación estadísticamente significativa entre los niveles de motivación extrínseca de las jugadoras de baloncesto y el país que representaron en el campeonato ( $x 2=12,995 ; p=0,043$ ).

Tabla 7. Niveles de motivación intrínseca y extrínseca según país de las jugadoras de baloncesto. Bogotá, 2018

\begin{tabular}{|c|c|c|c|c|c|c|c|c|}
\hline \multirow{2}{*}{ País } & \multicolumn{4}{|c|}{ Motivación intrínseca } & \multicolumn{4}{|c|}{ Motivación extrínseca } \\
\hline & Alta & No alta & $x^{2}$ & $\mathbf{p}$ & Alta & No alta & $x^{2}$ & $\mathbf{p}$ \\
\hline Argentina & 7 & 5 & 9,315 & 0,157 & 2 & 10 & 12,995 & $0,043^{*}$ \\
\hline Chile & 12 & 0 & & & 9 & 3 & & \\
\hline Colombia & 10 & 2 & & & 5 & 7 & & \\
\hline Ecuador & 10 & 2 & & & 6 & 6 & & \\
\hline Paraguay & 8 & 3 & & & 3 & 8 & & \\
\hline Perú & 11 & 1 & & & 4 & 9 & & \\
\hline Venezuela & 9 & 1 & & & 7 & 4 & & \\
\hline Total & 67 & 14 & & & 36 & 47 & & \\
\hline
\end{tabular}

* Significativo $a=0.05$

Fuente: los autores.

\section{Niveles de no motivación según país de las jugadoras}

Los resultados del cruce entre país y no motivación muestran, por una parte, un 13,4 \% de no motivación. Al discriminarla por países se observa que todos los equipos participantes en el torneo de baloncesto tienen al menos una jugadora que reveló no motivación, pero que en Paraguay (27,2 \%), Venezuela (20 \%) y Ecuador (16,7 \%) hay el 
mayor porcentaje de no motivación de todos los países. No obstante, no hay relación entre la no motivación y el país

$$
\left(x^{2}=3,648 ; p=0,779\right) \text {. }
$$

Tabla 8. Niveles de no motivación según País de las jugadoras de baloncesto. Bogotá, 2018

\begin{tabular}{lccccc}
\hline \multirow{2}{*}{ País } & \multicolumn{5}{c}{ No motivación } \\
\cline { 2 - 5 } & Presente & Ausente & & $\mathbf{X}^{\mathbf{2}}$ & $\mathbf{p}$ \\
\hline Argentina & 1 & 11 & 12 & 3,468 & 0,748 \\
\hline Chile & 1 & 11 & 12 & \\
\hline Colombia & 1 & 11 & 12 & \\
\hline Ecuador & 2 & 10 & 12 & \\
\hline Paraguay & 3 & 8 & 11 & \\
\hline Perú & 1 & 12 & 13 & \\
\hline Venezuela & 2 & 8 & 10 & \\
\hline Total & 11 & 71 & 83 & \\
\hline
\end{tabular}

Fuente: los autores.

\section{Discusión}

Este estudio tuvo como objetivo analizar la motivación tanto intrínseca como extrínseca y la no motivación en siete equipos nacionales de baloncesto femenino que participaron en el Encuentro Sudamericano Femenino en 2018, el cual tuvo lugar en Tunja, Boyacá (Colombia). El estudio se realizó con 86 mujeres deportistas de alto rendimiento pertenecientes a los equipos nacionales de baloncesto, competencia que incluyó Argentina, Chile, Colombia, Ecuador, Paraguay, Perú y Venezuela.

Con relación a la motivación en general, se comprobó que los datos, en comparación con las teorías de tipo mecanicistas, muestran la existencia de una gran influencia de los factores ambientales en la presencia de conductas que direccionan tal comportamiento (Hellin, 2010). La preocupación que orientó este estudio ayudó a conocer las diferencias más significativas entre países, en cuanto a la presencia de conductas motivacionales del tipo extrínseco versus intrínseco, en tanto que la primera se ancla a factores de tipo ambiental previamente mencionados y la segunda está más relacionada a componentes de la autodeterminación (Guzmán et al., 2013). 
En efecto, en cuanto a la motivación intrínseca, se encontró que los niveles de motivación en la mayoría de las basquetbolistas son altos, siendo Chile el país que se destaca por encima de los demás debido a que la totalidad del equipo se ubica estadísticamente en un nivel alto de motivación, mientras que las jugadoras de Argentina y Paraguay fueron las que representaron los menores porcentajes de alto nivel de motivación intrínseca de todos los países participantes en el torneo sudamericano de baloncesto. Perú, Colombia, Ecuador y Venezuela también se destacaron con niveles altos de motivación intrínseca; sin embargo, estas diferencias no son estadísticamente significativas (Guzmán-Luján y Carratalá-Deval, 2006).

La motivación intrínseca parece ser, en consecuencia, un componente anclado al compromiso deportivo como factor determinante para la participación activa en el baloncesto; ello implica además, que las condiciones internas de las jugadoras en cuanto al disfrute, características personales y otro tipo de factores que son de tipo interno del ser humano ayudan a revelar índices altos en la totalidad de los países participantes del estudio, sin diferencias estadísticamente significativas (Área de Alto Rendimiento Deportivo, 2008).

Del mismo modo, en cuanto a la motivación extrínseca, el equipo de Argentina fue el que mostró menores porcentajes de jugadoras con alto nivel de motivación extrínseca y Chile el mayor porcentaje, seguido de Venezuela (Balaguer et al., 2007). Se observa entonces una tendencia de las basquetbolistas argentinas, peruanas, paraguayas y colombianas a tener bajos niveles de motivación extrínseca, en tanto que las chilenas y venezolanas propendieron a tener altos niveles de motivación extrínseca (Esteve et al., 2005).

Ecuador no mostró ninguna tendencia, ya que la distribución según nivel fue paritaria; por tanto, hay suficiente evidencia empírica para afirmar que el nivel de motivación extrínseca está relacionado de manera significativa con el país al que pertenece cada selección de baloncesto (Martín et al., 2007).

La motivación extrínseca en el baloncesto puede depender de múltiples factores entre los cuales se pueden señalar: relación con las compañeras, relación con el entrenador, relación con los padres de familia, incentivos, tipo de práctica deportiva, lesiones, hábitos, responsabilidad y compromiso de equipo, percepción de las rivales, percepción de la tribuna, problemas de tipo familiar, personal, social, académico, entre otros (Girela, 2005).

Puede además estar relacionado con los recursos del equipo, la percepción del control, la autoconfianza de las compañeras, el éxito y el fracaso del equipo y de las jugadoras de manera individual, la influencia que ejercen los entrenadores, condiciones de preparación física, condiciones de salud física y otros factores ambientales 
que pueden intervenir en experiencias de tipo positivo y negativo que pueden estar afectando de manera significativa la motivación extrínseca (Buceta, 1999b).

A pesar de que la motivación intrínseca en los equipos de baloncesto de los siete países se destaque como alta, la motivación extrínseca en niveles bajos de algunos de ellos puede afectar de manera variada los motivos por los cuales una jugadora decida mantenerse o abandonar la práctica del baloncesto, la presencia de habilidades de afrontamiento, por ejemplo, puede ser decisiva en este fenómeno (Baquero y Limón, 1999).

Consecutivamente, se encontró que hay bajas proporciones de no motivación entre las jugadoras de baloncesto: de cada diez jugadoras, dos tienen no motivación. Además de esta baja prevalencia en la muestra, los resultados no permiten apoyar la existencia de relación entre la no motivación y el país (Aguirre y González, 2014).

En este sentido, Baquero y Limón (1999) afirman que la motivación intrínseca y extrínseca van ligadas, así que, progresivamente una afecta a la otra a partir de los refuerzos positivos que incentivan el impulso por hacer algo, guardando relación con las necesidades afectivas (Villamizar, Acosta, Cuadros y Montoya, 2009). Sin embargo, la no motivación está relacionada con la insatisfacción y ausencia tanto de MI como de ME; por lo tanto, los resultados al mostrar niveles altos de MI y ME ponen en evidencia una baja prevalencia de la no motivación, ya que la existencia de la una supone la ausencia de la otra, independientemente de si es extrínseca o intrínseca (Escartí y Cervelló, 1994).

También destaca que las selecciones de Chile y Venezuela son las que tienen altos niveles de motivación intrínseca y extrínseca, en tanto que Argentina fue el país que reveló menor proporción de estas motivaciones (Moreno et al., 2009). Los datos aportan suficiente evidencia para afirmar que los niveles de motivación extrínseca están relacionados con el país de procedencia de la selección, pero que la motivación intrínseca es independiente del país, lo que significa que es probable que la práctica deportiva no tenga diferencias estadísticamente significativas, entre la motivación que lleva al sujeto a realizar una acción por su propio interés y que está más influenciada por recompensas externas (Garita, 2006).

La psicología deportiva orientó este estudio en la medida en que permitió comprender acciones sobre el comportamiento deportivo de una muestra en particular, con una creciente relevancia para la disciplina, en tanto que ayuda a desarrollar y fortalecer de forma continua un nuevo ámbito de intervención (Méndez et al., 2016). Esto representa la consecución de resultados óptimos en cuanto a la competencia deportiva se refiere, por cuanto el tema motivacional en la psicología de la actividad física y del deporte es fundamental, debido a que permite el desarrollo de la autoeficacia, 
el establecimiento de metas, esfuerzo y habilidades de afrontamiento que orientan el comportamiento hacia el logro de objetivos buscando la prevención de recaídas psicológicas (Huilcapi et al., 2017).

A manera de conclusión, es importante señalar que la MI y la ME se presentaron en altos niveles en general; sin embargo, la no motivación puso en evidencia que al menos una jugadora de la totalidad de los equipos que participaron en este estudio se encuentra en ausencia de MI y ME con los porcentajes más altos en Paraguay (27,2 \%), Venezuela (20 \%) y Ecuador (16,7 \%) (López, 2011). Entonces, cabe destacar que es necesario intervenir desde la psicología deportiva este fenómeno en particular, ya que puede desencadenar en el futuro resultados inesperados de afectación tanto particular como grupal (Alfaro, 2010).

En cuanto a las limitaciones del estudio, vale la pena resaltar para futuras investigaciones la exploración de datos adicionales a la motivación deportiva como, por ejemplo, número de juegos ganados o puntajes obtenidos durante la competencia, que pudieran indicar cierta asociación entre la motivación y el rendimiento deportivo (Martínez et al., 2008).

Asimismo, la implementación de estrategias psicológicas, que resulte útil para el aumento de la motivación en las jugadoras de alto rendimiento de baloncesto femenino latinoamericano con un especial énfasis en los países Argentina y Perú. Además de los resultados de este estudio, se recomienda indagar a profundidad factores asociados a la ausencia de altos niveles de motivación intrínseca y extrínseca por profesionales de la salud mental deportiva, con el fin de mitigar ciertos efectos negativos que podría generar la ausencia de motivación a diferencia de Chile y Venezuela, cuyas puntuaciones favorables en motivación sobresalen por encima de los demás países latinoamericanos (Montero et al., 2015).

\section{Referencias}

Aguirre L, H. y González, J. (2014). Factores motivacionales, variables deportivas y sociodemográficas en deportistas universitarios. Tesis Psicológica, 9(1),130-145. https://www.redalyc.org/ pdf/1390/139031679011.pdf

Alfaro, E. (2010). Las mujeres y el deporte: evolución y situación actual. Seminario permanente Mujer y deporte. Universidad Politécnica de Madrid.

Área de Alto Rendimiento Deportivo. (2008). Área de Alto Rendimiento Deportivo. Cultura, Ciencia y Deporte, 3(8), 29-67. https://www.redalyc.org/pdf/1630/163028253003.pdf 
Bakhsh, Q. B. (2008). Effects of job satisfaction on employees motivation \& turn over intentions. (From review of the related literature). Journal of Managerial Sciences, 2(1), 1-21.

Balaguer, I., Castillo, I. y Duda, J. (2007). Propiedades psicométricas de la escala de motivación deportiva en deportistas españoles. Revista Mexicana de Psicología, 24(2), 197-207.

Baquero, R., y Limón, M. (1999). Teorías del aprendizaje. Universidad Nacional de Quilmes.

Blanco, I. y Buceta, J.M. (1993). Intervención psicológica con el Equipo Nacional Olímpico de Hockey Hierba Femenino. Revista de Psicología del Deporte, (3) 87-99.

Buceta, J. M. (1999a). El papel del baloncesto en el desarrollo y la formación de los jóvenes. https:// palestraweb.com/documents/01_BALONCESTO.pdf

Buceta, J. M. (1999b). Variables psicológicas relacionadas con el rendimiento deportivo. UNED.

Broo, N., Ballart, P., Juan, B., Valls, A. y Latinjak, A. (2012). Motivación situacional y estado afectivo en clases dirigidas de actividad física. Motricidad. European Journal of Human Movement, 29,147-158. https://dialnet.unirioja.es/descarga/articulo/4775366.pdf.

Cox, R. (2009). Psicología del deporte. Conceptosy sus aplicaciones. Editorial Médica Panamericana.

Cruz, J. (1997). Psicología del deporte. Síntesis.

De Diego, S. y Sagredo, C. (1992). Jugar con ventaja: las claves psicológicas del éxito deportivo. Alianza Editorial.

Deci, E., y Ryan, R. (1990). A motivational approach to self: Integration in personality. Nebraska Symposium on Motivation, 38, 237-288.

Dosil, J. y Caracuel, J.C. (2003). Psicología aplicada al deporte. En J. Dosil (ed.), Ciencias de la Actividad Física y el Deporte, (pp. 155-186). Síntesis.

Escartí, A. y Cervelló, E. (1994). La motivación en el deporte. En I. Balaguer (Ed.), Entrenamiento psicológico en deporte: principios y aplicaciones (pp. 61-90). Albatros Educación.

Esteve R, J., Musitu O, G. y Murillo, M. (2005). Autoconcepto físico y motivación deportiva en chicos y chicas adolescentes. La influencia de la familia y de los iguales. Escritos de PsicologíaPsychological Writings, (7), 82-90. https://www.redalyc.org/pdf/2710/271020873008.pdf 
Flores, G. y Ruiz, F. (2009). Relación de algunos correlatos biológicos y demográficos con la práctica físico-deportiva en estudiantes universitarios. El caso de la Universidad de Guadalajara, México. Revista Internacional de Ciencias del Deporte, 5(14), 59-80.

García-Sandoval, J. y Caracuel-Tubio, J. (2007). La motivación hacia la práctica deportiva en adolescentes mexicanos: Inicio, mantenimiento y abandono. Revista Iberoamericana de Psicología del Ejercicio y el Deporte, 2(1), 41-60.

García, J., Caracuel, J. y Ceballos, O. (2014). Motivación y ejercicio físico deportivo: una añeja relación. Revista Internacional de Ciencias Sociales y Humanidades, SOCIOTAM, XXIV (1), 71-88.

Garita, E (2006). Motivos de participación y satisfacción en la actividad física, el ejercicio físico y el deporte. MHSalud, 3(1),1-16. https://www.redalyc.org/pdf/2370/237017528002.pdf

Giménez, F. (2004). Aspectos teóricos y prácticos de la iniciación al baloncesto. Editorial Wanceulen.

Girela, M, J. (2005). Las diferencias de género existentes en el conocimiento profesional docente del alumnado de la Facultad de Ciencias de la Actividad Física y del Deporte de la Universidad de Granada. Apunts Educación Física y Deportes, 3(81), 104. https://www.raco.cat/index.php/ ApuntsEFD/article/view/300948

Gómez, C. (2019). Relación entre las dimensiones de personalidad según el P-IPG y la calidad de vida laboral percibida. Acta Colombiana de Psicología, 22(1), 230-238.

González, A. y Ríos, M. (1999). Crecer con el deporte. En G. Nieto y E. J. Garcés de Los Fayos (coords.), Psicología de la Actividad Física y el Deporte, Tomo II (pp. 456-469). Sociedad Murciana de Psicología de la Actividad Física y el Deporte.

González, C., Guevara, Y., Jiménez, D. y Alcázar, R. (2018). Relación entre asertividad, rendimiento académico y ansiedad en una muestra de estudiantes mexicanos de secundaria. Acta Colombiana de Psicología, 21(1), 116-127. https://doi.org/10.14718/ACP.2018.21.1.6

Gotzent, C. y González, C. (1993). Socialización. En L. Pérez (ed.), 10 palabras claves en superdotados (pp. 215-244). Verbo Divino.

Guillén, F. (2007). Psicología de la educación física y el deporte. Editorial Wenceulen S.L.

Gutiérrez, M. (2000). Actividad física, estilos de vida y calidad de vida. Revista de Educación Física, (77), 5-14. 
Guzmán, J., Macagno, L. y Imfeld, F. (2013). La motivación de los entrenadores deportivos: un estudio desde la teoría de la autodeterminación. Cuadernos de Psicología del Deporte, 13(1), 37-49.

Guzmán-Luján, J. y Carratalá-Deval, V. (2006). Mediadores psicológicos y motivación deportiva en judocas españoles. RICYDE. Revista Internacional de Ciencias del Deporte, I/(5), 1-11.

Hellin, H. (2010). Hábitos físico-deportivos en la región de Murcia: implicaciones para la elaboración del currículum en el ciclo formativo de actividades físico-deportivas. [Tesis de doctorado, Universidad de Murcia].

Hernández, R., Fernández, C., y Baptista, P. (2014). Metodología de la investigación (6ª edición). Editorial Mc Graw Hill.

Huilcapi, M., Castro, G. y Jácome, G. (2017). Motivación: las teorías y su relación en el ámbito empresarial. Dominio de las Ciencias, 3(2), 311-333.

López, N. (2011). Relación entre factores motivacionales y niveles de ansiedad en porristas en precompetencia. International Journal of Psychological Research, 4(1),80-91. https://www. redalyc.org/pdf/2990/299022819010.pdf

Martín, J., Núñez, J., Navarro, J., Leite, M., Almirón, M. y Glavinich, N. (2007). Propiedades psicométricas de la versión española de la escala de motivación deportiva en Paraguay. Revista Mexicana de Psicología, 24(1),43-52. https://www.redalyc.org/pdf/2351/235119266007.pdf

Martínez, R., Molinero, O., Jiménez, R., Salguero, A., Tuero, C. y Márquez, S. (2008). La motivación para la práctica en la iniciación al fútbol: influencia de la edad/categoría competitiva, el tiempo de entrenamiento y la relación con el entrenador. Apunts Educación Física y Deportes, (93),46-54. https://www.redalyc.org/articulo.oa?id=551656932007

Méndez, A., Cecchini, J. A. y Fernández, J. (2016). Pasión por el deporte, actividad física vigorosa y satisfacción con la vida. Psicología del Deporte, 25(1), 73-79.

Montgomery, L. N., Schmerr, N., Burdick, S., Forster, R. R., Koenig, L., Legchenko, A., Ligtenberg, S., Miège, C., Miller, O. L. y Solomon, D. K. (2017). Investigation of Firn Aquifer Structure in Southeastern Greenland Using Active Source Seismology. Frontiers in Earth Science, 5, 10. https://doi.org/10.3389/feart.2017.00010

Montero, I. y León, O. G. (2007). A guide for naming research studies in Psychology. International Journal of Clinical and Health Psychology, 7(3), 847-862. 
Montero, C., González, D., Moreno, J., Carratalá, V. y Cervelló, E. (2015). Motivación, estado de ánimo y flow en judocas de élite. Revista Mexicana de Psicología, 32(2),101-112. https://www.redalyc. org/pdf/2430/243045364001.pdf

Moreno, J., Zomeño, T., Marín, L., Cervello, E. y Ruiz, L. (2009). Variables motivacionales relacionadas con la práctica deportiva extraescolar en estudiantes adolescentes de educación física. Apunts Educación Física y Deportes, (95), 38-43. https://www.redalyc.org/articulo. oa?id=5516/551656931006

Moreno, J., Cervelló, E. y González, D. (2006). Motivación autodeterminada y flujo disposicional en el deporte. Anales de Psicología, 22 (2), 310-317.

Moreno, J., Pavón, A., Gutiérrez, M. y Sicilia-Camacho, A. (2005). Motivaciones de los universitarios hacia la práctica físico-deportiva. Revista Internacional de Medicina y Ciencias de la Actividad Física y del Deporte / International Journal of Medicine and Science of Physical Activity and Sport, 5(19), 154-165.

Mújica, A., Guido, P. y Gutiérrez, R. (2012). Factores motivacionales y de capital cultural que inciden en el comportamiento lector en estudiantes mexicanos de nivel medio superior de diferente estrato social. Acta Colombiana de Psicología 15(1), 21-31.

Muyor, J., Águila, C., Sicilia, A. y Orta, A. (2009). Análisis de la motivación autodeterminada en usuarios de centros deportivos. Revista Internacional de Medicina y Ciencias de la Actividad Física y del Deporte, 9(33), 67-80.

Pelletier, L. G., Fortier, M. S., Vallerand, R. J., Tuson, K. M., Briere, N. M. y Blais, M. R. (1995). Toward a new measure of intrinsic motivation, extrinsic motivation, and amotivation in sports: The Sport Motivation Scale (SMS). Journal of Sport \& Exercise Psychology, 17(1), 35-53.

Puig, N. (1992). Itineraris esportius de la població juvenil. Una aproximació sociològica. En VII Jornades de l'Associació Catalana de Psicologia de l'Esport (ACPE), (pp. 11-35). Barcelona: ACPE. Congreso llevado a cabo en Barcelona, España noviembre de 1992.

Pulido, J., Sánchez, D., Sánchez, P., Leo, F. y García, T. (2016). Influencia de la formación de los entrenadores sobre la motivación de los deportistas. Revista Internacional de Medicina y Ciencias de la Actividad Física y del Deporte, 16(64), 685-702.

Pulido, J., Sánchez, D., González, I., Amado, D., Montero, C. y García, T. (2015). Adaptación y validación de un cuestionario para valorar la motivación en el contexto deportivo. Cuadernos de Psicología del Deporte, 15(3), 17-26. 
26 Motivación deportiva en jugadoras de baloncesto de alto rendimiento de las selecciones nacionales de Argentina, Chile, Colombia, Ecuador, Paraguay, Perú y Venezuela

Valdés, H. (1990). La preparación psicológica del deportista. Mente y rendimiento humano. INDE Publicaciones.

Villamizar, M., Acosta, J., Cuadros, J. y Montoya, J. (2009). Factores motivacionales de los futbolistas profesionales, como deportistasy clientes internos en Boyacá, Colombia. International Journal of Psychological Research, 2(2),148-157. https://www.redalyc.org/pdf/2990/299023513008. $\mathrm{pdf}$ 